\title{
GENETIC ALGORITHM FOR OPTIMIZATION OF THE AEDES AEGYPTI CONTROL STRATEGIES
}

\author{
Helenice O. Florentino ${ }^{1 *}$, Daniela R. Cantane ${ }^{1}$, Fernando L.P. Santos ${ }^{1}$, \\ Célia A. Reis ${ }^{2}$, Margarida V. Pato ${ }^{3}$, Dylan Jones ${ }^{4,5}$, Marianna Cerasuolo ${ }^{5}$, \\ Rogério A. Oliveira ${ }^{1}$ and Luiz G. Lyra ${ }^{1}$
}

Received October 16, 2017 / Accepted June 29, 2018

\begin{abstract}
Dengue Fever, Zika and Chikungunya are febrile infectious diseases transmitted by the Aedes species of mosquito with a high rate of mortality. The most common vector is Aedes aegypti. According to World Health Organization outbreaks of mosquito-borne illnesses are common in the tropical and subtropical climates, as there are currently no vaccines to protect against Dengue Fever, Chikungunya or Zika diseases. Hence, mosquito control is the only known method to protect human populations. Consequently, the affected countries need urgently search for better tools and sustained control interventions in order to stop the growing spread of the vector. This study presents an optimization model, involving chemical, biological and physical control decisions that can be applied to fight against the Aedes mosquito. To determine solutions for the optimization problem a genetic heuristic is proposed. Through the computational experiments, the algorithm shows considerable efficiency in achieving solutions that can support decision makers in controlling the mosquito population.
\end{abstract}

Keywords: Optimization models, genetic algorithms, healthcare operational research.

\section{INTRODUCTION}

Tropical illness has increased significantly and the risk of Dengue Fever, Zika and Chikungunya can potentially affect half of the world's population. Viruses transmitted by the Aedes species of mosquito cause these illnesses, which in most cases are similar to flu. Sometimes they lead to

\footnotetext{
*Corresponding author.

${ }^{1}$ Departamento de Bioestatística - IB, UNESP, Bairro Rubião Júnior, 18618-670 Botucatu, SP, Brasil.

E-mails: helenice.silva@unesp.br; daniela.cantane@unesp.br; fernando.pio@unesp.br; rogerio.oliveira@unesp.br; luizgustavolyra@yahoo.com.br

2 Departamento de Matemática - FC, UNESP, Bauru, SP, Brasil. E-mail: celia.reis@ unesp.br

${ }^{3}$ ISEG and CMAFCIO, Universidade de Lisboa, Portugal. E-mail: mpato@iseg.ulisboa.pt

${ }^{4}$ Centre for Operational Research and Logistics, University of Portsmouth, UK. E-mail: dylan.jones@ port.ac.uk

$5^{5}$ Department of Mathematics, University of Portsmouth, UK. E-mail: marianna.cerasuolo@port.ac.uk
} 
major health problems, and in some countries are one of the leading causes of death in children and adults, as detailed in [20].

There are five Dengue serotypes, denoted by DEN-I, DEN-II, DEN-III, DEN-IV and DEN-V. Zika is an infection caused by the ZIKV and Chikungunya is caused by the Chikungunya virus (CHIKV). These viruses are members of the virus family Flaviviridae and the genus Flavivirus, ([14], [18]).

Humans are the primary host of these viruses, but they also circulate between other primates. The mosquitoes receive the virus by biting an infected human; after eight to 10 days, the virus is already in the mosquito saliva, and the infection is transmitted to the other people they bite. Symptoms appear in humans from three to 14 days after an infective bite, ([15], [17]).

Only the female mosquitoes bite for blood, because it is used in the maturation process of eggs. After blood feeding the female lays the eggs on damp surfaces in humanized areas likely to temporarily flood. Females produce on average 100 to 200 eggs per batch, with about five separated batches of eggs during a lifetime. In this way the mosquitoes spread, promoting the rapid diffusion of diseases ([4]).

WHO warns the world population regarding the risks of these tropical diseases, because at the moment there are no vaccines neither effective treatments for Dengue Fever, Chikungunya and Zika. Prevention is the only way to fight the problem. Prevention works by controlling the main factor of infection, the mosquitoes of Aedes aegypti species ([4], [8]).

The control of mosquitoes can be carried out in all phases of their life: the aquatic phase (egg, larva and pupa) and the terrestrial phase (adult mosquitoes). In Brazil SUCEN (Superintendence of Endemic Disease Control) generally uses two types of control for mosquitoes' aquatic and terrestrial phase: physical control, performed by public health officials and residents by removing breeding areas; and chemical control, i.e., applying insecticides. Another type of control, which at the moment is in an experimental stage, is the biological control using sterilized male mosquitoes. In this case, sterile male mosquitoes are produced in a laboratory. They are unable to generate offspring, as they are bombarded with gamma radiation. This technique is known as Sterile Insect Technique (SIT), and has been shown to be effective in controlling agricultural pests ([9]).

Some studies have been performed in order to determine optimal controls of the Aedes mosquito population. In Thomé et al. [17] a mathematical model is proposed to describe the dynamics of the mosquito population using chemical control with insecticide, and biological control with sterile male mosquitoes. Here the aim is to minimize the costs of insecticide application, production of irradiated mosquitoes and the number of fertilized female mosquitoes. The solution of the model was determined by applying Pontryagin's Maximum Principle. The study of Florentino et al. [4] is devoted to an optimization model similar to the one in [17], and is solved using a multi-objective genetic algorithm. Dias et al. [2] used the multiobjective model proposed in [4] to study the alternated step-size control and trade-off solutions were obtained using an evolutionary multiobjective algorithm. The results obtained in [2] are quite similar to the ones in [4]. 
All these cited works discuss the difficulties encountered when applying the methodology they propose, specially the difficulty of applying the results in practice.

The paper of Wijaya and Soewono [21] discusses an optimal control model using chemical controls with insecticide on aquatic and terrestrial phases of the development of the mosquito. A version of the gradient-based method is presented to minimize the objective functional with respect to the control variable. Numerical results are presented to analyze the reduction of the population. Mishra and Gakkhar [13] developed a nonlinear model involving human and vector populations aiming to study the effects of awareness and mosquito control on dengue epidemic. The authors present a complete analysis of the dynamics of this model.

In [16] an optimal control model of Dengue epidemics is presented, where the objective function represents the costs of medical treatment of infected people, and of educational and sanitation campaigns. The problem is solved within the classical optimal control theory and by nonlinear programming following discretization. The results show a decrease in the number of infected mosquitoes and individuals.

An optimal control problem for vector dengue transmission was modeled in [1]. The authors included physical control in their model, by considering mosquito repellent for adults and children. The objective function includes the costs for treatment of infected people, and of reducing the drop-out rates. Numerical results of the optimal controls, for the case of epidemic prevention and outbreak reduction strategies, are presented.

Zhang et al. [24] proposed a delayed model for the dynamics and control of a mosquito population aiming to determine an integrated strategy that includes chemical, mechanical and biological (using SIT) controls. The authors investigate different control regimes under varying environmental temperature by means of numerical simulations.

The search for better ways to control mosquito-borne diseases is intense, and much research has been done in this area (namely, [6], [12], [22]).

In this paper, we propose an optimization model to investigate the intensity of three types of mosquito control - physical (removal of breeding), chemical (by spreading insecticide) and biological (by introducing sterilized mosquito males) - that simultaneously minimize the damage of the insecticide, the costs of production and insertion of the sterilized mosquitoes in nature, the number of fertilized females (because they are responsible for transmission of diseases), the effect of the insecticide on sterilized mosquitoes and the amount of breeding (because this can be an expensive and complex process). The model presented in this research includes an objective function that aggregates these five different objectives. By using this model, the decision makers may adapt the weights and generate a solution that is in line with their preferences. We present a description of a genetic algorithm designed to solve this model. Computational experiments and conclusions are presented in the last section. 


\section{MATHEMATICAL METHODOLOGIES}

\subsection{Mosquito population dynamics}

The life cycle of the mosquito includes two phases: the aquatic phase (egg, larva and pupa stages) and the terrestrial phase (adult stage). In the aquatic phase, at time $t$, the mosquito population density is given by $A(t)$. These mosquitoes become adults at a per capita rate $\gamma$, with a proportion $r$ of females and $(1-r)$ of males. At time $t$, the population of adult mosquitoes comprises three segments: the females before mating, with density $I(t)$, the natural males, $M(t)$, and the fertilized females, $F(t)$, as shown in Figure 1. The insects are assumed to mate (once) with rate $\beta$. The per capita oviposition rate is given by $\phi(1-A(t) / C)$, where $\phi$ is the intrinsic oviposition rate and $\mathrm{C}$ is the carrying capacity depending on the amount of available nutrients and space. The per capita mortality rates of $A(t), I(t), M(t)$ and $F(t)$ are given by $\mu A, \mu I, \mu M$ and $\mu F$, respectively.

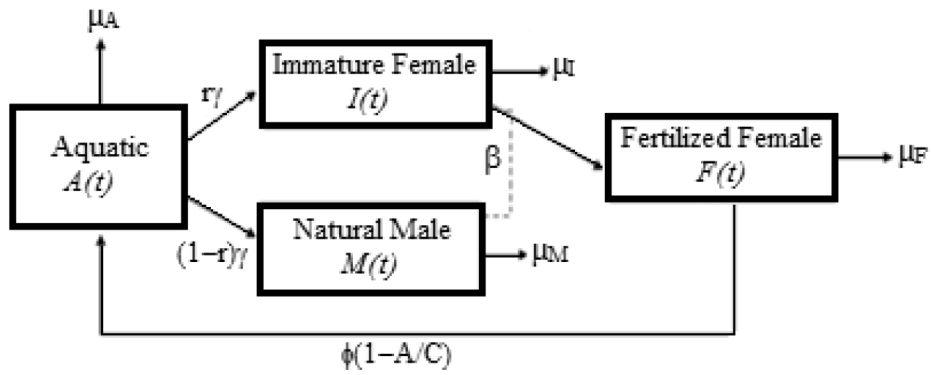

Figure 1 - Non-controlled population dynamics of the mosquitoes (adapted from Florentino et al. (2014)).

The mathematical model that describes this non-controlled population dynamics (MPD1) is given by:

$$
\begin{aligned}
\frac{d A}{d t} & =\phi\left(1-\frac{A}{C}\right) F-\gamma A-\mu_{A} A \\
\frac{d I}{d t} & =r \gamma A-\beta I-\mu_{I} I \\
\frac{d F}{d t} & =\beta I-\mu_{F} F \\
\frac{d M}{d t} & =(1-r) \gamma A-\mu_{M} M .
\end{aligned}
$$

This basic model represents the dynamics of an Aedes mosquito population (Esteva and Yang 2006).

Figure 2 illustrates the evolution of the mosquito population without control for 300 days, starting with a population of only a few individuals $(A(0)=2.9 \mathrm{e}-3, I(0)=2.4310 \mathrm{e}-7, F(0)=$ $8.3286 \mathrm{e}-8, M(0)=2.9727 \mathrm{e}-6)$. It can be observed that all segments of the population are increasing and they stabilize at very high values. 


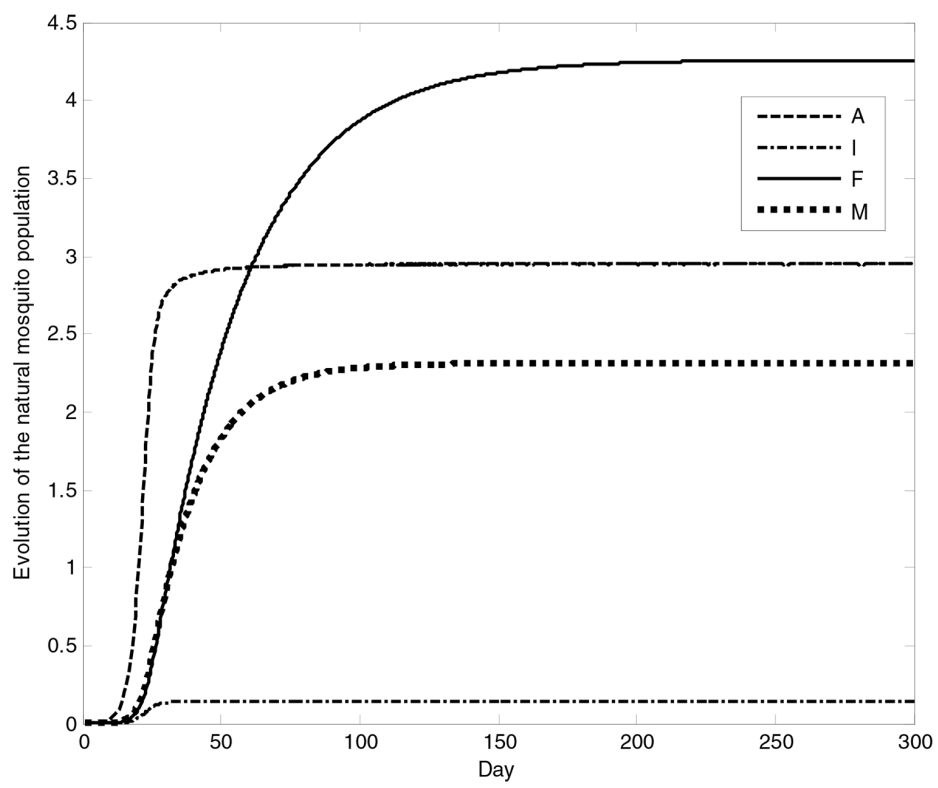

Figure 2 - The evolution of the mosquito population without control for 300 days.

Three ways of controlling the mosquito population in the aquatic and adult phases when it reaches a high density in a specific area have been studied: chemical control using insecticide, biological control using sterilized male mosquitoes, either irradiated or transgenic ([9]), and physical control using removal of breeding.

As observed in [4], biological control has the disadvantage of a high cost of production and spread of mosquitoes in the targeted area. Chemical control has a lower cost and a great efficiency for mosquito population reduction. However, increasing the amount of insecticide causes resistance of future mosquito generations, hence the need for extra products, and the consequent environmental damage. Physical control using withdrawal of breeding inside houses and in other places involves community participation and hence requires appropriate educational measures. Consequently, this process has high social and economic costs. Simultaneous use of chemical, biological and physical controls can keep a reasonable efficacy of chemical control without the need of incurring the large costs associated with the sole use of physical and biological controls.

Florentino et al. [4] review important studies on optimal control of mosquitoes and extend a mathematical model proposed in [3] embedding chemical and biological controls. Their model considers two control variables, $u_{1}(t)$ associated with the insecticide spread at time $t$ (reduction rate for mosquitoes' density), and $u_{2}(t)$ related to the density of sterile male insects placed in the environment, at time $t$. Two additional population segments are considered there: sterile mosquitoes, with density $S(t)$, and unfertilized females, $U(t)$, which are the females that mate with sterile males and are removed from the system. A female from $I(t)$ meets a natural male with probability $M(t) /(M(t)+S(t))$ and gets fertilized at a rate $\beta$. A female from $I(t)$ meets 
a sterile male from $S(t)$ with probability $p S(t) /(M(t)+S(t))$, where $p, 0 \leq p \leq 1$, is the proportion of sterile males placed in the area. Sterilization can reduce the mating rate to $q \beta$, with $0 \leq q \leq 1$. The insecticide acts only in the adult stage of the mosquito and not in the aquatic phase. Figure 3 illustrates the dynamics of this system.

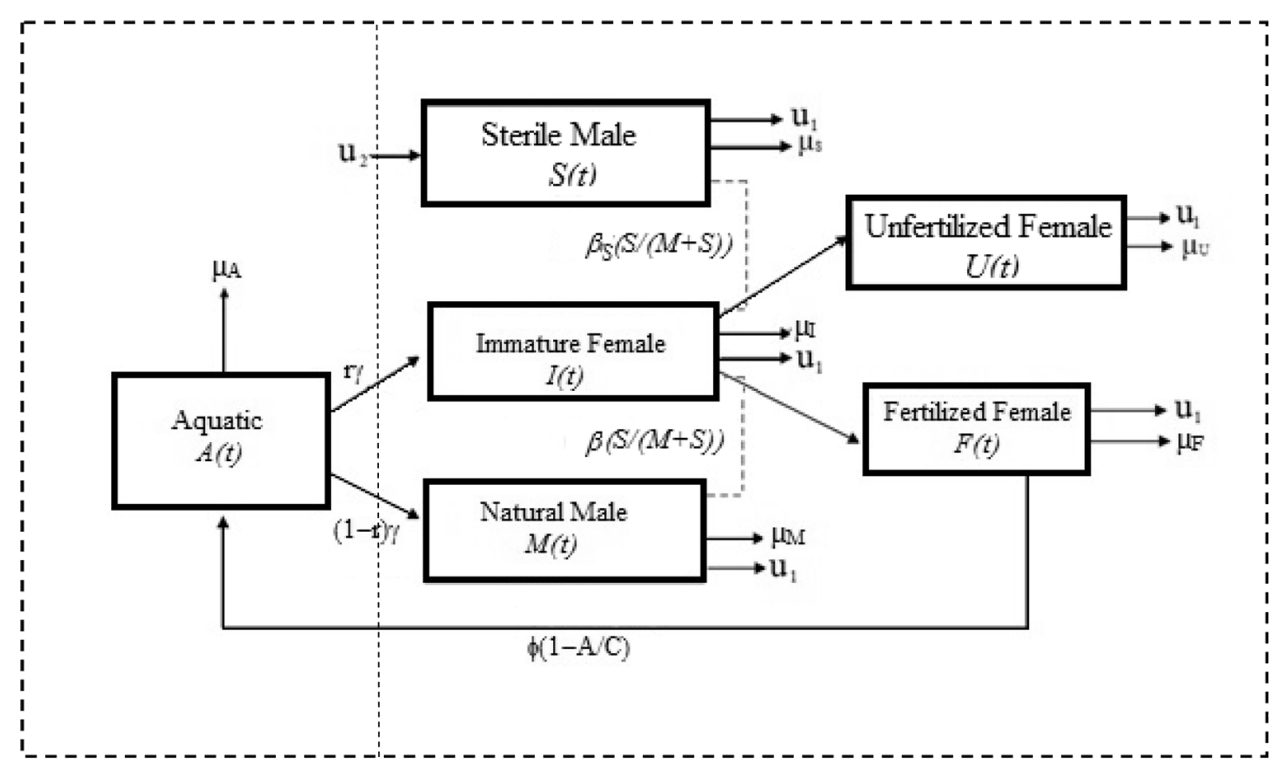

\section{Aquatic phase}

Terrestrial phase

Figure 3 - Population dynamics of mosquitoes with biological control by sterile males (source: Florentino et al. (2014)).

In this study we propose the addition of the physical control to the described process, by defining a new control variable $u_{3}(t)$ that represents the reduction rate of density of mosquitoes in the aquatic phase as a result of the breeding removal at time $t$. The mathematical model representing this dynamics (MPD2) is as follows (based on [6] and [17]):

$$
\begin{aligned}
\frac{d A}{d t} & =\phi\left(1-\frac{A}{C}\right) F-\gamma A-\mu_{A} A-u_{3} A \\
\frac{d I}{d t} & =r \gamma A-\frac{\beta M I}{M+S}-\frac{\beta_{S} S I}{M+S}-\mu_{I} I-u_{1} I \\
\frac{d F}{d t} & =\frac{\beta M I}{M+S}-\mu_{F} F-u_{1} F \\
\frac{d M}{d t} & =(1-r) \gamma A-\mu_{M} M-u_{1} M \\
\frac{d S}{d t} & =u_{2}-\mu_{S} S-u_{1} S .
\end{aligned}
$$


The equilibrium conditions for system MPD2 with $u_{3}=0$ correspond to the worst possible situation from the standpoint of controlling disease and are used as initial conditions for the system MPD2's state variables:

$$
\left\{\begin{array}{l}
A(0)=\frac{C(R-1)}{R} \\
I(0)=\frac{r \gamma A(0)}{\mu_{I}+\beta} \\
F(0)=\frac{\left(\gamma+\mu_{A}\right) C A(0)}{\phi(C-A(0))} \\
M(0)=\frac{(1-r) \gamma A(0)}{\mu_{M}} \\
S(0)=0
\end{array}\right.
$$

where

$$
R=\frac{\phi r \gamma \beta}{\left(\gamma+\mu_{A}\right)\left(\beta+\mu_{I}\right) \mu_{F}}
$$

defines the average number of secondary females produced by a single female. When $R>1$ a stable endemic equilibrium occurs ([3], [17]). More details on properties of similar models can also be found in [3] and [16].

\subsection{Optimization model}

Considering that the above defined variables, $u_{1}=u_{1}(t), u_{2}=u_{2}(t)$ and $u_{3}=u_{3}(t)$, are the variables associated, respectively, with insecticide (chemical control), with sterilized mosquitoes to be placed in nature (biological control), and with removal of breeding (physical control), the optimization model (MPDO) follows:

$$
\text { Minimize } J=\frac{1}{2} \int_{0}^{T}\left(u_{1}^{2}+u_{2}^{2}+F^{2}+\left(u_{1} S\right)^{2}+u_{3}^{2}\right) d t
$$

subject to

$$
\begin{aligned}
& \frac{d A}{d t}=\phi\left(1-\frac{A}{C}\right) F-\gamma A-\mu_{A} A-u_{3} A \\
& \frac{d I}{d t}=r \gamma A-\frac{\beta M I}{M+S}-\frac{\beta_{S} S I}{M+S}-\mu_{I} I-u_{1} I \\
& \frac{d F}{d t}=\frac{\beta M I}{M+S}-\mu_{F} F-u_{1} F \\
& \frac{d M}{d t}=(1-r) \gamma A-\mu_{M} M-u_{1} M \\
& \frac{d S}{d t}=u_{2}-\mu_{S} S-u_{1} S \\
& F \leq F_{f i x}, \text { for all } t \geq t_{f i x}
\end{aligned}
$$




$$
u_{1} \geq 0, u_{2} \geq 0, u_{3} \geq 0
$$

where $J=J\left(u_{1}, u_{2}, u_{3}\right), A=A(t), I=I(t), F=F(t), M=M(t), S=S(t), u_{1}=u_{1}(t)$, $u_{2}=u_{2}(t)$ and $u_{3}=u_{3}(t)$ for $t \in[0, T]$, and $T$ represents the duration of the period of control application. The functional $J$ aggregates the five proposed objective functions $u_{1}^{2}, u_{2}^{2}, u_{3}^{2}, F^{2}$ and $\left(u_{1} S\right)^{2} . F_{f i x}$ is a desired output of $F(t)$ for $t$ greater or equal to the time parameter $t_{f i x}$ (this constraint forces the optimization method to work as a closed-loop control system, hence $F_{f i x}$ can be understood as a reference output of $F(t)$ for a time $t_{f i x}$ ) and the initial conditions for the ordinary differential system MPD2 are as defined at the end of Section 2.1.

The MPDO is developed to determine levels of mosquito population control from a solution $\left(u_{1}(t), u_{2}(t), u_{3}(t)\right)$ that simultaneously minimizes the insecticide to be used, the quantity of sterilized mosquitoes to be inserted into the natural environment, the quantity of fertilized females in the population, the effect of the insecticide on sterilized mosquitoes $\left(u_{1} S\right)$, and the investment in removal of breeding in houses and other places, at each time $t$. Parameter $R$ is used to generate the initial conditions. The last functional constraint of the model is included to keep the female population at low levels from a predefined time onwards.

Thomé et al. [17] and Florentino et al. [4] solve a similar optimal control problem by using only a chemical control (insecticide) and a biological control (sterilized mosquitoes). The objective of this problem is to minimize the cost.

In [17] an approach based on the Pontryagin's Maximum Principle was used. This methodology involves an adjoint system to impose the optimality conditions on the state and control variables. The solution of this expanded system (the original system with an adjoint system and transversality conditions) gives the optimal controls. According to [17], the high number of variables in the expanded system and the instability of the adjoint variables make the system difficult to solve. The authors proposed a methodology based on the analytic continuation method which reformulates the problem into one-parameterized family of problems. However, this methodology is not suitable for the introduction of additional control variables to work with specific control functions (for instance, constants or piecewise constants, which are more appropriate to fit to reality), and also to account for the dynamical system constraints (for instance, to force the output to follow a desired reference).

In [4] a bi-objective genetic algorithm (GA) for the problem presented in [17] was developed, but only chemical and biological controls were considered. In this approach, it is possible to work with specific control functions and to add a state-reference output. However, this multi-objective methodology produces a set of potentially Pareto optimal solutions which can be difficult to interpret by the health care decision makers as no single recommended solution is given and is also less efficient from the computational perspective. Moreover, these authors did not consider physical control, which has great importance in tropical disease prevention because it is the cheapest and most accessible control for the low-income population.

In this paper we develop a GA that will be presented in the next sub-section. The GA is chosen over the above methodologies due to its flexibility in incorporating the extra conditions detailed 
above, its pragmatic nature that allows for the generation of realistic solutions in reasonable computational time ([7]) and its ability to generate a single recommended solution with a good fitness that can be put forward to the health care decision makers.

\subsection{Genetic algorithm}

As mentioned above, the complexity of classical techniques of optimal control and the significant numerical difficulties of their computational development, along with the high cardinality of the set of final solutions of any bi-objective genetic algorithm supported the option by an aggregated objective genetic algorithm to solve the MPDO, referred to as GA_AEDES. Genetic algorithms are very efficient in searching good quality solutions, for a wide variety of optimization problems, because they do not possess many of the limitations found in traditional methods ([7]). In fact, GAs do not require a well behaved objective function and they can be tailored to produce feasible solutions, thus being adaptable to optimization problems with a wide variety of objective functions and constraints. They have also the advantage of determining a discretized solution that is well suited for real world application as in the specific case analyzed in this paper.

The algorithm GA_AEDES explores the properties of the problem in so far as its solutions are represented by matrices containing the values for the three proposed controls, chemical, biological and physical, and the algorithm also includes a state-reference output and other features that are described in the remainder of this Section. The fitness $\left(F_{\text {ind }}\right)$ of each chromosome, an MPDO solution, is determined from the value of the objective function of MPDO on that solution $\left(J_{i n d}\right)$, and also from the sum of the violations of its constraints $\left(P_{\text {ind }}\right)$.

This method is characterized by the following features (an explanation of genetic algorithm features may be found in the seminal GA text of ([7]):

\section{Algorithm GA_AEDES}

\section{Generation $\leftarrow 1$;}

2. Generate the initial population $\left(P_{\text {Generation }}\right)$ :

$n$ randomly generated individuals, each one associated with a solution of MPDO and characterized by a chromosome represented by a $3 \times(T+1)$ dimensional matrix, as shown by Figure 4a;

3. Evaluate the fitness for each individual:

each individual ind is evaluated by its fitness $F_{\text {ind }}$ given by

$$
F_{\text {ind }}=1 /\left(J_{\text {ind }} P_{\text {ind }}\right), \quad \text { ind }=1,2, \ldots, n
$$

where $J_{\text {ind }}$ is the value of the functional in MPDO and $P_{\text {ind }}$ penalizes the infeasibility of the individual ind;

4. Copy and reserve $K$ elite individuals:

in the first generation, the $K$ best solutions of the population are stored in the elite set; 


\begin{tabular}{c|c|c|c|c|c|c|}
\multicolumn{1}{c}{} & \multicolumn{1}{c}{1} & \multicolumn{1}{c}{$\ldots$} & \multicolumn{1}{c}{$i$} & $\ldots$ & $T$ \\
\cline { 2 - 7 }$u_{1}(t)$ & $u_{1}(0)$ & $u_{1}(1)$ & $\ldots$ & $u_{1}(i)$ & $\ldots$ & $u_{1}(T)$ \\
\cline { 2 - 7 }$u_{2}(t)$ & $u_{2}(0)$ & $u_{2}(1)$ & $\ldots$ & $u_{2}(i)$ & $\ldots$ & $u_{2}(T)$ \\
\cline { 2 - 7 }$u_{3}(t)$ & $u_{3}(0)$ & $u_{3}(1)$ & $\ldots$ & $u_{3}(i)$ & $\ldots$ & $u_{3}(T)$ \\
\cline { 2 - 7 } & & &
\end{tabular}

(a)

|| Randomly chosen crossover point

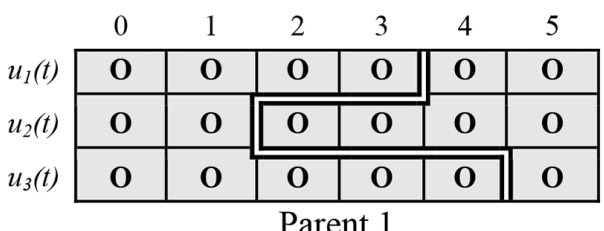

\begin{tabular}{|c|c|c|c|c|c|c|}
\hline & 0 & 1 & 2 & 3 & 4 & 5 \\
\hline$u_{1}(t)$ & $\mathbf{X}$ & $\mathbf{X}$ & $\mathbf{X}$ & $\mathbf{X}$ & $\mathbf{X}$ & $\mathbf{X}$ \\
\hline$u_{2}(t)$ & $\mathbf{X}$ & $\mathbf{X}$ & $\mathbf{X}$ & $\overline{\mathbf{X}}$ & $\mathbf{X}$ & $\mathbf{X}$ \\
\hline$u_{3}(t)$ & $\mathbf{X}$ & $\mathbf{X}$ & $\bar{X}$ & $\mathbf{X}$ & $\bar{X}$ & $\mathbf{X}$ \\
\hline
\end{tabular}

Parent 2

\begin{tabular}{|c|c|c|c|c|c|c|}
\hline & 0 & 1 & 2 & 3 & 4 & 5 \\
\hline$u_{1}(t)$ & $\mathbf{O}$ & O & O & 0 & $\mathbf{X}$ & $\mathbf{X}$ \\
\hline$l_{2}(t)$ & O & O & $\mathbf{X}$ & $\mathbf{X}$ & $\mathbf{X}$ & $\mathbf{X}$ \\
\hline$u_{3}(t)$ & $\mathbf{O}$ & 0 & 0 & 0 & 0 & $\mathbf{X}$ \\
\hline
\end{tabular}

\begin{tabular}{|c|c|c|c|c|c|c|}
\hline & 0 & 1 & 2 & 3 & 4 & 5 \\
\hline$u_{1}(t)$ & $\mathbf{X}$ & $\mathbf{X}$ & $\mathbf{X}$ & $\mathbf{X}$ & $\mathbf{O}$ & 0 \\
\hline$u_{2}(t)$ & $\mathbf{X}$ & $\mathbf{X}$ & $\mathbf{O}$ & O & $\mathbf{O}$ & O \\
\hline$u_{3}(t)$ & $\mathbf{X}$ & $\mathbf{X}$ & $\mathbf{X}$ & $\mathbf{X}$ & $\mathbf{X}$ & O \\
\hline
\end{tabular}

Offspring

(b)

Figure 4 - (a) Chromosome structure for daily control during $T+1$ days. (b) Crossover: reproduction operator ( $T=5$ days).

\section{Repeat for Generation}

5.1 Select parents from population ( $\mathrm{P} \%$ of $n$ ) using the roulette wheel technique [7];

5.2 Perform crossover on parents - one-point crossover performed in each chromosome row, as shown in Figure 4b;

5.3 Perform mutation of population with probability $\mathrm{Pm}$ (see description below);

5.4 Determine fitness for each new individual generated by crossover and mutation, as discussed in step 3; (somente passo 3)

5.5 Create an intermediate population $P_{\text {int }}=P_{\text {Generation }} \cup$ \{individuals generated by crossover $\} \cup$ \{individuals generated by mutation $\}$;

5.6 Generation $\leftarrow$ Generation +1 ;

5.7 Create a new population $\left(P_{\text {Generation }}\right)$ by retaining the $n$ best fitted individuals from $P_{\text {int }}$;

5.8 Update the elite set - the $K$ best solutions of the new population are merged within the elite set, and the $K$ best among them are stored as elite for the next generation; 
6. Until Generation $=G$ (stopping criterion: maximum number of generations);

7. Output: the best solutions of the elite set.

The individuals of the initial population are randomly built according to a uniform distribution in $[0,1]$, in $[0,10 M(0)]$ and in $[0,1]$, respectively, for $u_{1}(t), u_{2}(t)$ and $u_{3}(t)$, and $t=0, \ldots, T$. As for the mutation operator, after selecting a chromosome with probability $P_{m}$, the genes to mutate are chosen, per chromosome row, according to a discrete uniform distribution in the range $[0, T]$. The value of each gene that has been selected to mutate is randomly generated (uniform distribution) in its respective interval.

More information about the genetic algorithm can be found in [7], [19] or [25].

\section{RESULTS}

The algorithm GA_AEDES was implemented with MATLAB 7.14.0.739 (R2012a) software, MATLAB ([11]), and ran on a micro-computer Dual Core i5-650 with 8 GB memory and 400 GB hard drive.

Table 1 shows the MPDO parameters for a particular case when the average local temperature is $25^{\circ} \mathrm{C}$, defined on the basis of studies in [10], [17] and [23]. All parameters are measured per day $\left(d^{-1}\right)$ except $r$. Note that, the parameter values were chosen in accordance with the climatic and environmental conditions of Brazil.

Table 1 - Model MPDO parameters.

\begin{tabular}{c|c|c|c|c|c|c|c|c|c|c}
\hline$\phi$ & $C$ & $\gamma$ & $r$ & $\beta$ & $\beta_{S}$ & $\mu_{A}$ & $\mu_{I}$ & $\mu_{F}$ & $\mu_{M}$ & $\mu_{S}$ \\
\hline 6.353 & 3 & 0.0941 & 0.5 & 0.927 & 0.5 & 0.0583 & 0.0202 & 0.0319 & 0.06 & 0.07 \\
{$[*]$} & {$[*]$} & {$[* * *]$} & {$[*]$} & {$[* * *]$} & {$[* *]$} & {$[* * *]$} & {$[* *]$} & {$[* *]$} & {$[*]$} & {$[*]$} \\
\hline
\end{tabular}

(Source: [*] Thomé et al. (2010), [**] Koiller et al. (2014) and [***] Yang et al. (2009)).

Table 2 presents the parameters used for GA_AEDES. $T$ is the duration of the period of the control application. For the initial set of experiments it is set equal to 120 days, representing a four month hot and rainy summer period in a country such as Brazil when Dengue, Chikungunya and Zika outbreak are prone to occur. In order to conduct a sensitivity analysis with respect to the outbreak interval, additional sets of experiments were constructed using instances with times equal to 60,130, 180 and 365 days, which are presented and discussed at further on in this Section. Parameter $G$ is the maximum number of generations, $n$ is the number of individuals in the population, $P$ is the penalty imposed on infeasible individuals (equal to 1 , otherwise), $K$ is the cardinality of the elite, $\mathrm{p} \%$ is the percentage of individuals to be selected for the mating pool (hence, the mating pool has $p n$ individuals), $P_{m}$ is the mutation probability. All parameters were calibrated with exhaustive experiments performed to choose the best value adapted to the problem. Finally, $F_{f i x}$ is the reference output for $F(t)$, and finally, $t_{f i x}$ is the respective time value. 
Table 2 - Parameters for GA_AEDES algorithm.

\begin{tabular}{c|c|c|c|c|c|c|c|c}
\hline$T$ & $G$ & $n$ & $P$ & $K$ & $p$ & $P_{m}$ & $t_{f i x}$ & $F_{f i x}$ \\
\hline 120 & 100 & 300 & $10^{6}$ & 50 & 0.70 & 0.05 & 60 & 0.30 \\
\hline
\end{tabular}

Standard techniques as in [5] are used to calculate the functional in MPDO and to solve the system of nonlinear ordinary differential equations MPD2.

The results of the computational experiments are summarized in the following paragraphs. After running the algorithm for 100 generations it always attained feasibility for all 300 individuals of the population. The best fitted solution in the final population is retained as the result of the algorithm.

In the 120-day research work seven cases are studied for evaluation of the methodology using: only chemical control; only biological control; only physical control; chemical and biological controls together; chemical and physical controls together; biological and physical controls together; and the three controls, chemical, biological and physical, together. The average CPU time was 15 minutes per run of each instance. The Figures 5-11 show the performance of the GA_AEDES for resolution of the MPDO measured by the speed of convergence along the generations of the algorithm and the capacity in reduction of the mosquito population.

Figure 5 shows the evolution of the GA_AEDES results when the algorithm is applied to MPDO using only chemical control $u_{1}$, where $J_{1}=J\left(u_{1}, u_{2}, u_{3}\right)$, and $u_{2}(t)$ and $u_{3}(t)$ are identically zero functions for all $t=0, \ldots, T$.

Figure 6 shows the evolution of the GA_AEDES results when using only biological control $u_{2}$, where $J_{2}=J\left(u_{1}, u_{2}, u_{3}\right)$, and $u_{1}(t)$ and $u_{3}(t)$ are identically zero functions for all $t=0, \ldots, T$.

Figure 7 shows the evolution of the GA_AEDES results when using only physical control $u_{3}$, where $J_{3}=J\left(u_{1}, u_{2}, u_{3}\right)$, and $u_{1}(t)$ and $u_{2}(t)$ are identically zero functions for all $t=0, \ldots, T$.

Figure 8 shows the evolution of the GA_AEDES results when using chemical and biological controls, $u_{1}$ and $u_{2}$, where $J_{12}=J\left(u_{1}, u_{2}, u_{3}\right)$, and $u_{3}(t)$ is an identically zero function for all $t=0, \ldots, T$.

Figure 9 shows the evolution of the GA_AEDES results when using chemical and physical controls, $u_{1}$ and $u_{3}$, where $J_{13}=J\left(u_{1}, u_{2}, u_{3}\right)$, and $u_{2}(t)$ is an identically zero function for all $t=0, \ldots, T$.

Figure 10 shows the evolution of the GA_AEDES results when using biological and physical controls, $u_{2}$ and $u_{3}$, where $J_{23}=J\left(u_{1}, u_{2}, u_{3}\right)$, and $u_{1}(t)$ is an identically zero function for all $t=0, \ldots, T$.

Figure 11 shows the evolution of the GA_AEDES results when using simultaneously chemical, biological and physical controls $\left(u_{1}, u_{2}\right.$ and $\left.u_{3}\right)$ where $J=J\left(u_{1}, u_{2}, u_{3}\right)$. 

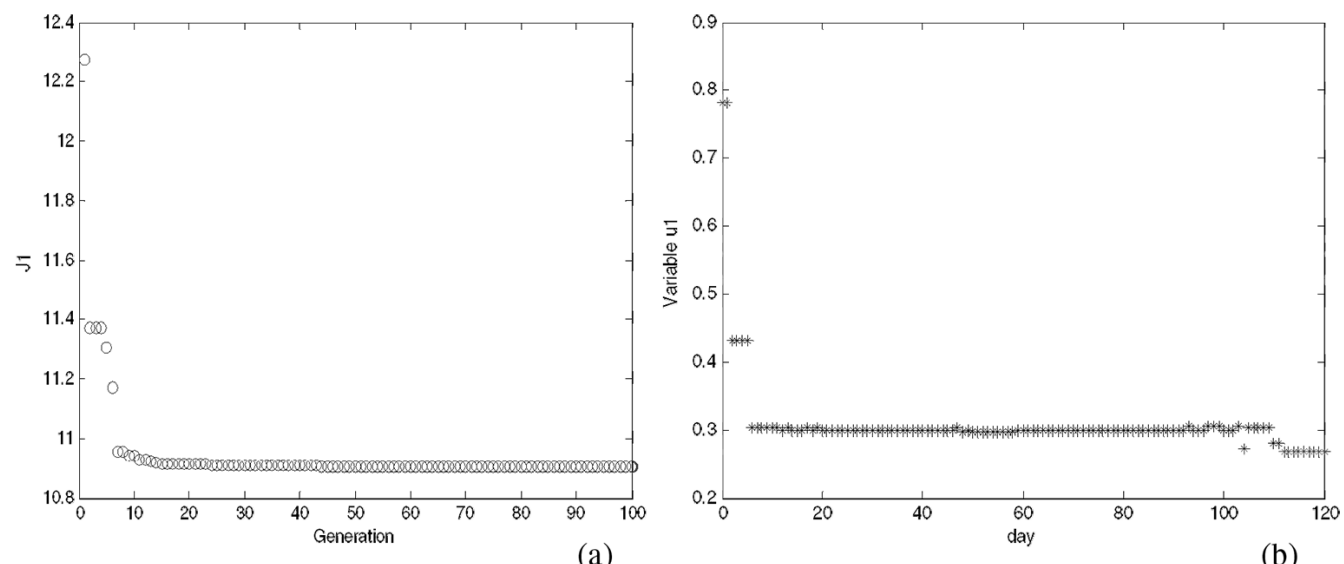

(a)

(b)

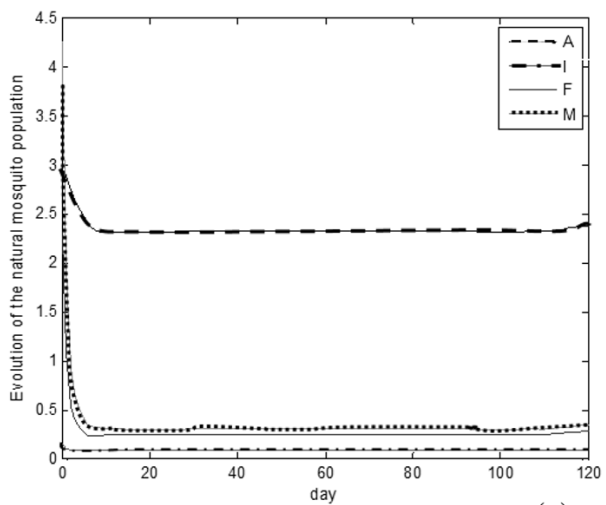

(c)

Figure 5 - Results of GA_AEDES algorithm using only $u_{1}$ control: (a) objective function value along the generations; (b) best value for control variable $u_{1}$; (c) evolution of natural mosquitoes population segments $A, I, F$ and $M$.

It can be observed that in Figures 5a-11a the GA_AEDES converged in the 5-15 generation range for all cases discussed. In the case of control with only insecticide (i.e. chemical control), (5b), the best solution obtained suggests the application of a high quantity of insecticide in the first days, then to gradually reduce the amount of insecticide for 5 days, up to $40 \%$ of its initial value, and finally to maintain this quantity in the remaining period. In the case of only controlling with sterilized males, (6b), the best solution suggests a high quantity of insects in the first 5 days and to reduce the quantity of these insects for about 80 days; and to use a low quantity in the remaining days. Using only physical control, (7b), the best solution suggests the withdrawal of a high quantity of breeding throughout the complete period. When any two of the three (or the three) controls are used simultaneously, the GA_AEDES solution suggests a decrease of the mentioned quantities, (8b-11b and 8c-11c). 

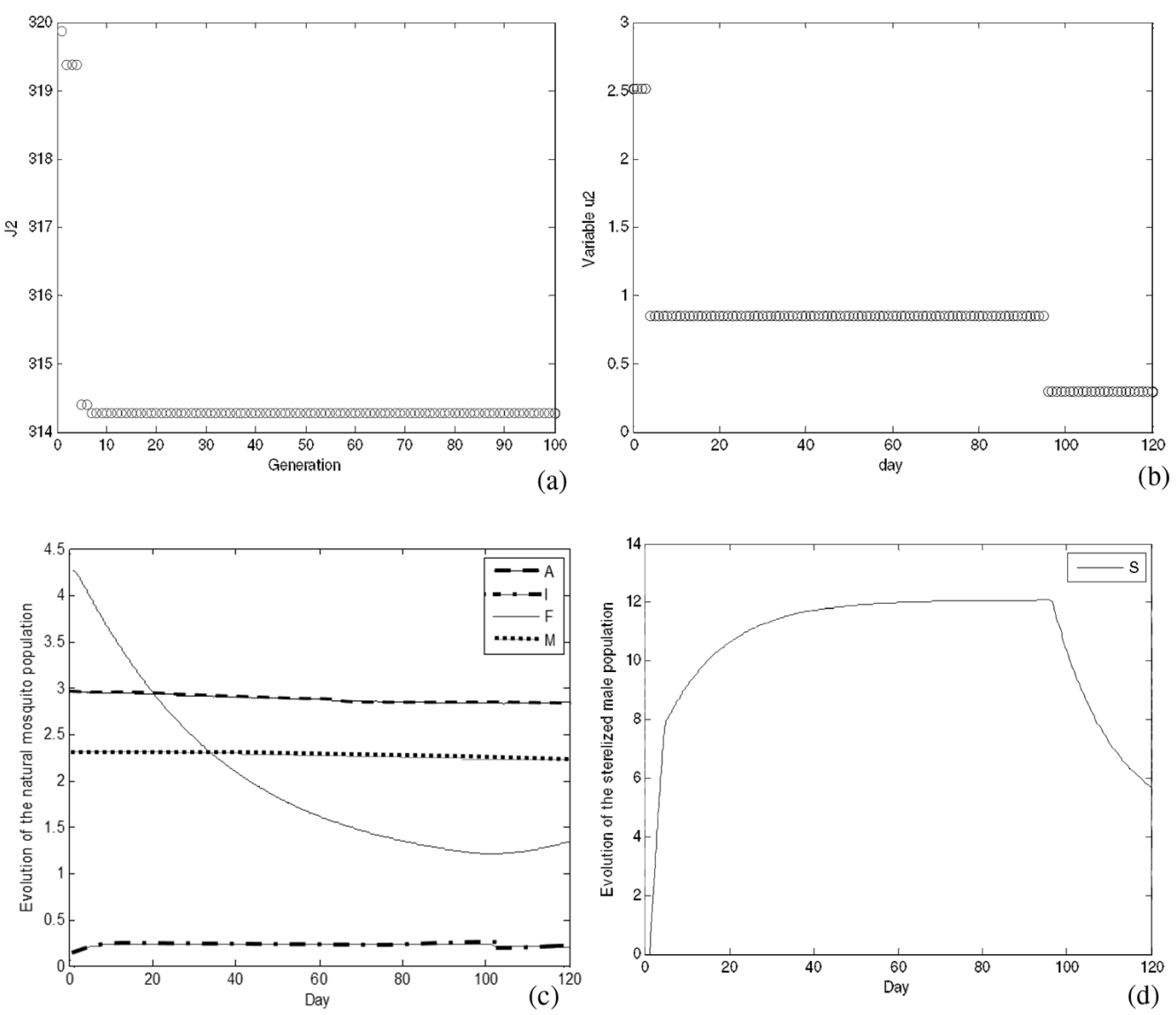

Figure 6 - Results of GA_AEDES algorithm using only $u_{2}$ control: (a) objective function value along the generations; (b) best value for control variable $u_{2}$; (c) evolution of natural mosquito population - segments $A, I, F$ and $M$; (d) evolution of sterilized mosquitoes population $S$.

A comparison of chemical and biological control techniques indicates that both controls are complementary, (8b-8c) and (11b-11c). This means that if we decide upon intensifying the spread of insecticide in the area attacked by Aedes mosquito, the quantity of sterile mosquitoes placed in the area can be reduced, and when deciding upon insecticide reduction, then more sterile mosquitoes must be used for controlling purposes. Similarly, a comparison of biological and physical controlling techniques indicates that these controls are complementary, (10b-10c) and (11c-11d).

Figures 5-11 show the evolution of the mosquito population segments under the seven cases solved by GA_AEDES. It is evident that there is a decrease of all segments of the population of natural mosquitoes during the period, for all cases that use insecticide (5c, 8d, 9d and 11e). For cases of the control without insecticide, the reduction is slower and the population of natural mosquitoes is only controlled at high levels (6c, 7c and 10d). This experiment indicates that the 

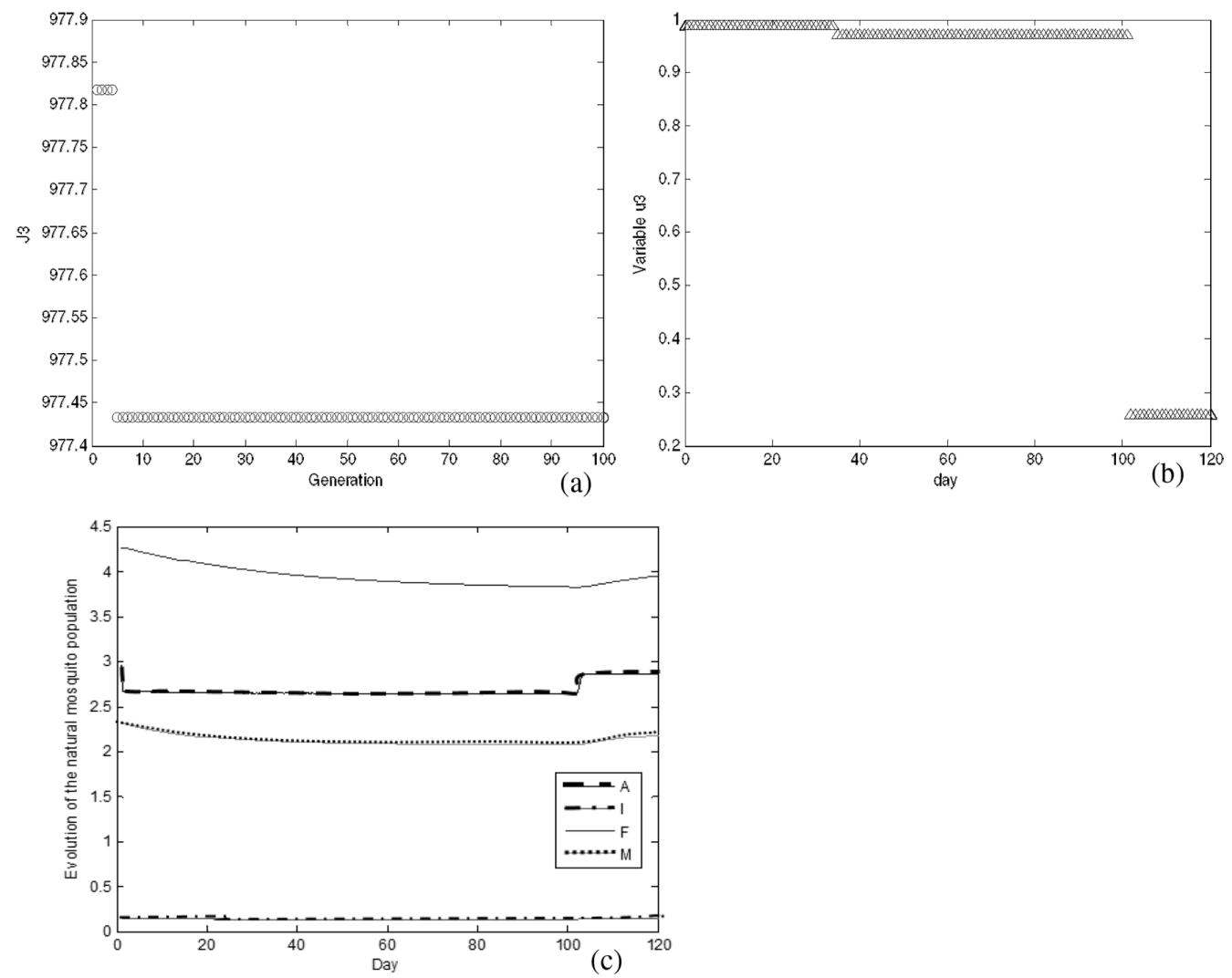

Figure 7 - Results of GA_AEDES algorithm using only $u_{3}$ control: (a) objective function value along the generations; (b) best value for control variable $u_{3}$; (c) evolution of natural mosquito population - segments $A, I, F$ and $M$.

control with insecticide is more effective for decreasing the number of mosquitoes than the other controls. The action of the insecticide on the mosquito is fast and causes death in an instant, this is the reason why the insecticide is the most used control in practice, but the health surveillance has warned about the risks of this to human and animal health and also insects can become resistant to the insecticide.

In Brazil, the insecticide is dispersed into the air in the form of a fog composed of mineral oil added insecticide, which kills the adult mosquito. It is applied in specific locations and hours during the outbreak of diseases (in the summer). Many problems have been reported regarding the use of this control. Our study shows that an adequate combination of chemical, biological and physical controls allows the reduction of insecticide application (Figure 11), thus improving the quality of the environment, and of animal and human life. Reduction in insecticide also reduces the possibility for the next generation of mosquitoes to acquire insecticide resistance. Another 

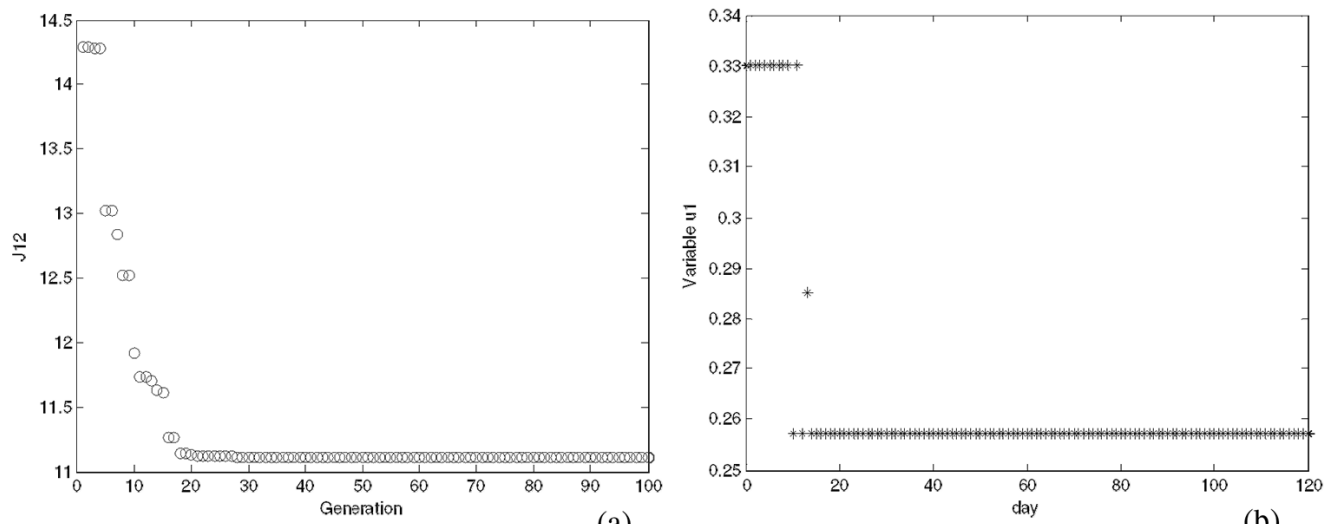

(a)

(b)
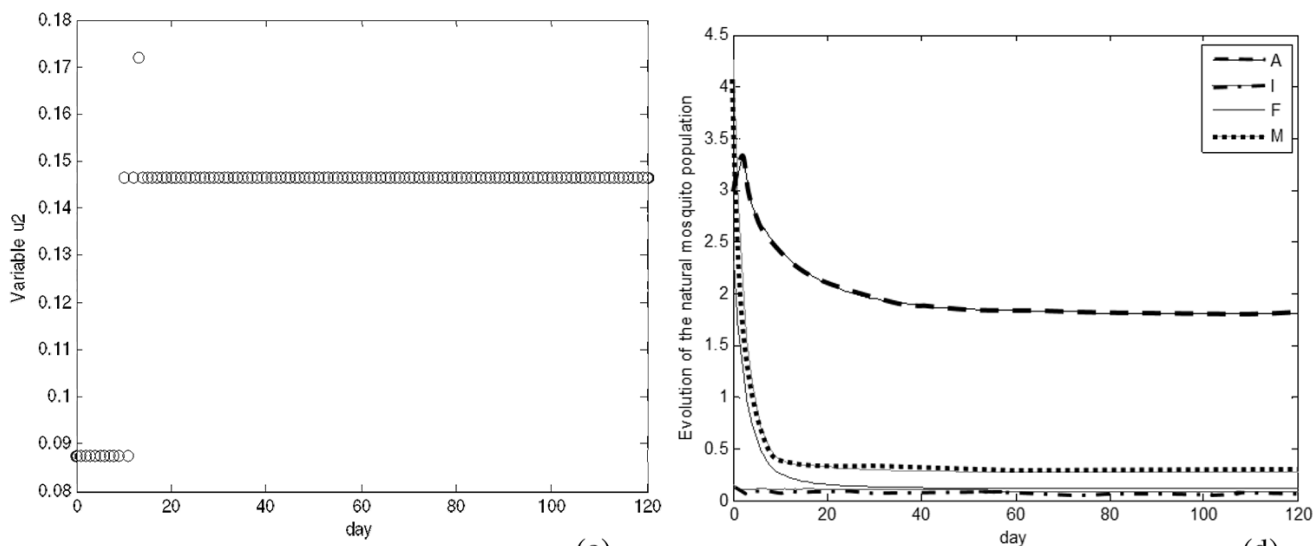

(c)

(d)

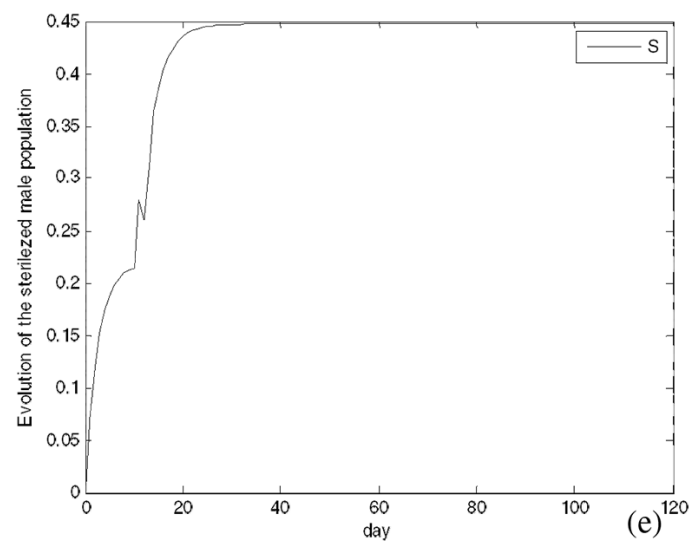

Figure 8 - Results of GA_AEDES algorithm when using $u_{1}$ and $u_{2}$ controls: (a) objective function value along the generations; (b) and (c) contain, respectively, the best $u_{1}$ and $u_{2}$ controls; (d) evolution of natural mosquito population - segments $M, F, I$ and $A$; (e) evolution of sterilized mosquito population $S$. 

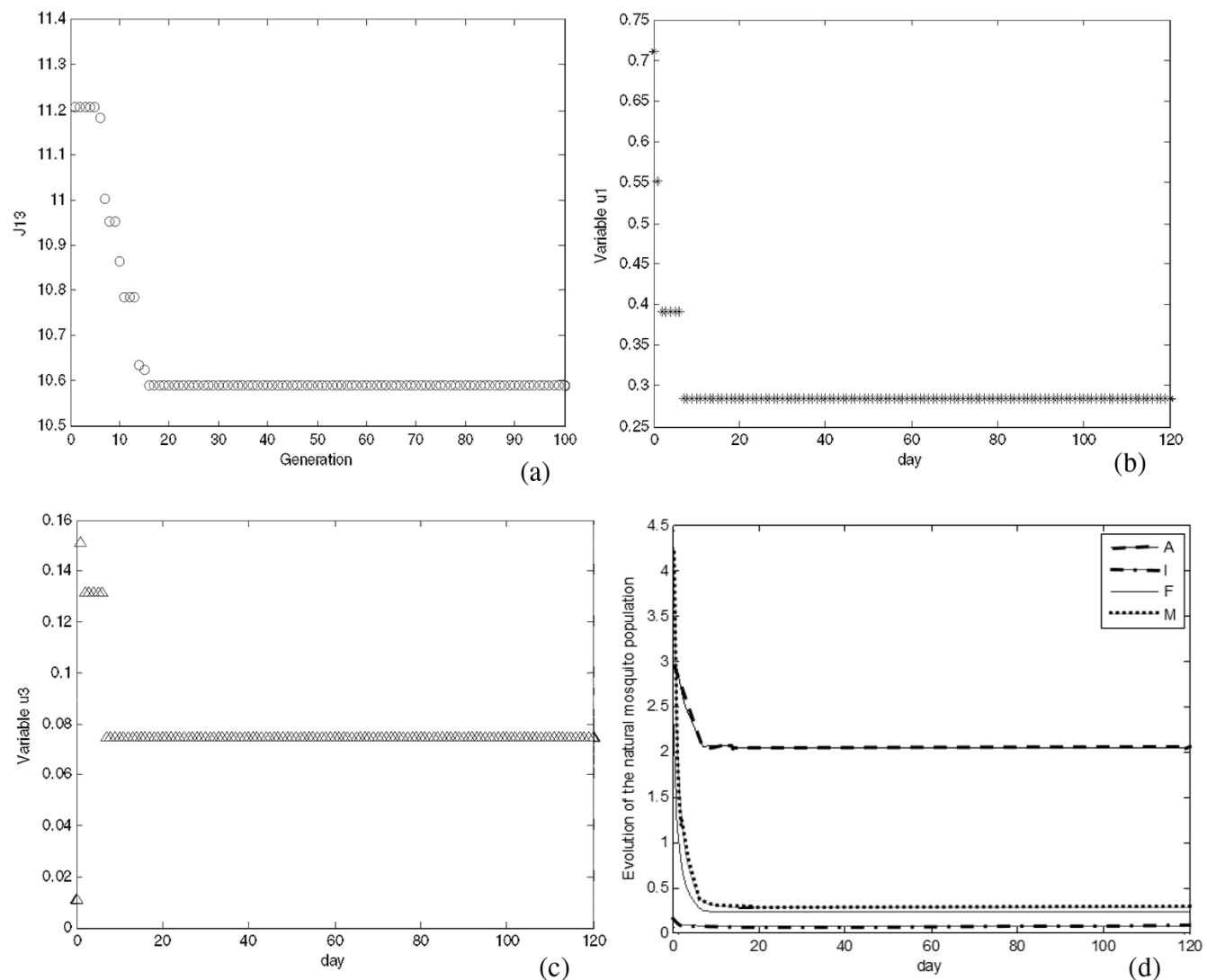

Figure 9 - Results of GA_AEDES algorithm when using $u_{1}$ and $u_{3}$ controls: (a) objective function value along the generations; (b) and (c) contain, respectively, the best $u_{1}$ and $u_{3}$ controls; (d) evolution of natural mosquito population-segments $M, F, I$ and $A$.

important advantage of the combination of these three controls is the large decrease in the population of natural mosquitoes, (Figure 11e), which minimizes the risk of diseases outbreak.

The summer in Brazil occurs in the first four months of the year (January-April), it is a hot and humid period presenting optimal conditions for the growth of the Aedes aegypti mosquito population, and represents the time of the year when the greatest number of mosquito transmitted diseases takes place. For this reason there is frequent need for sanitary surveillance authorities to apply vector controls in this period. In this context and in order to conduct sensitivity analysis with respect to the duration of an outbreak, we also investigate some instances in which control was applied during part or throughout the year, beginning in the summer period, during which the population is at high levels.

The instances described in Table 3 were analyzed in order to study the performance of the methodology on the varying time period instances. 


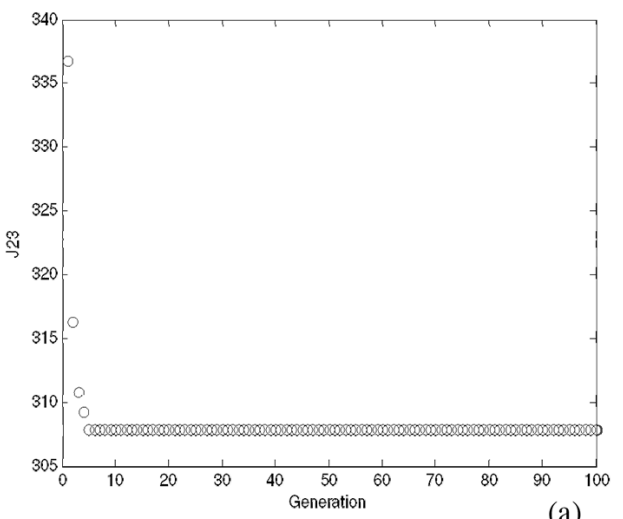

(a)

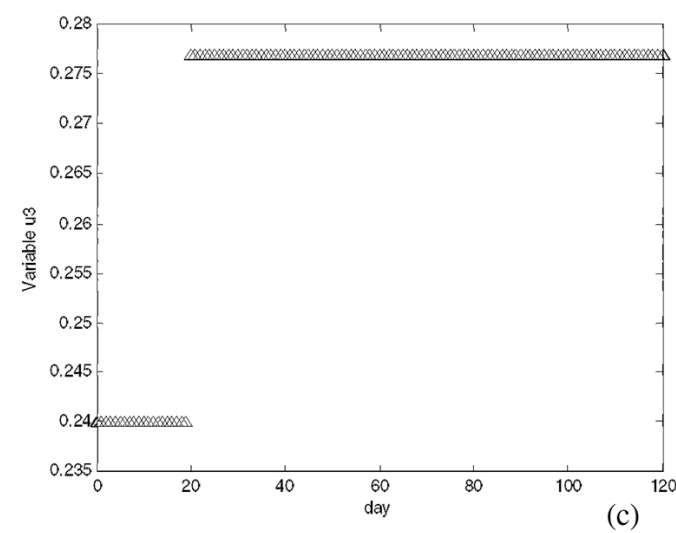

(c)

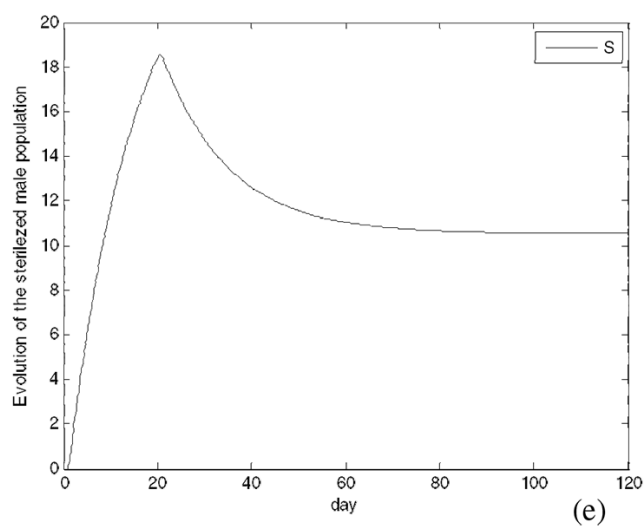

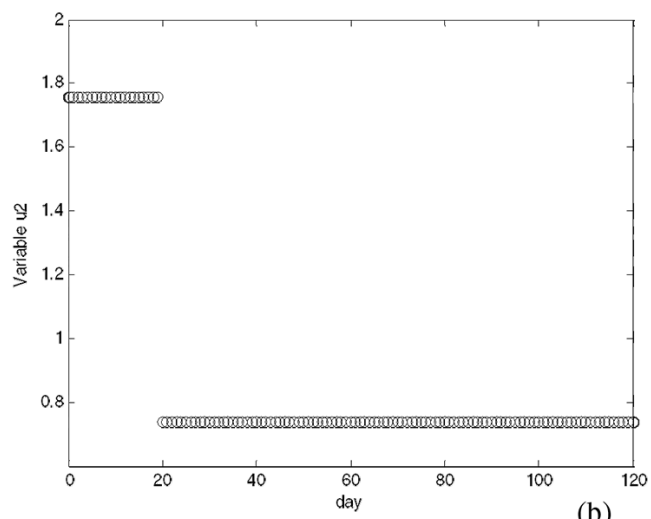

(b)

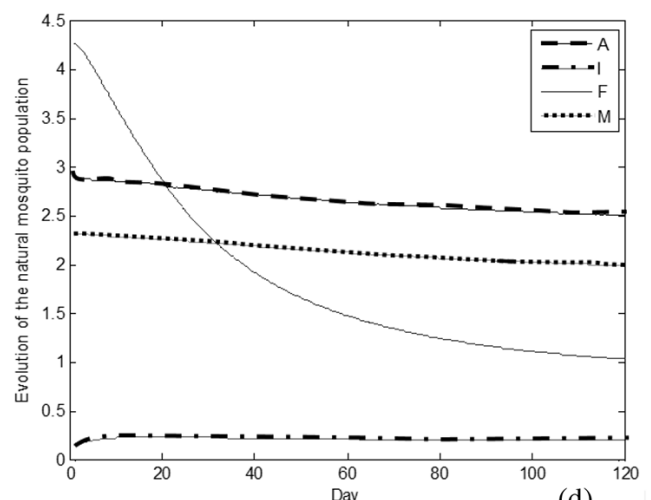

(d)

Figure 10 - Results of GA_AEDES algorithm when using $u_{2}$ and $u_{3}$ controls: (a) objective function value along the generations; (b) and (c) contain, respectively, the best $u_{2}$ and $u_{3}$ controls; (d) evolution of natural mosquito population - segments $M, F, I$ and $A$; (e) evolution of sterilized mosquito population $S$. 

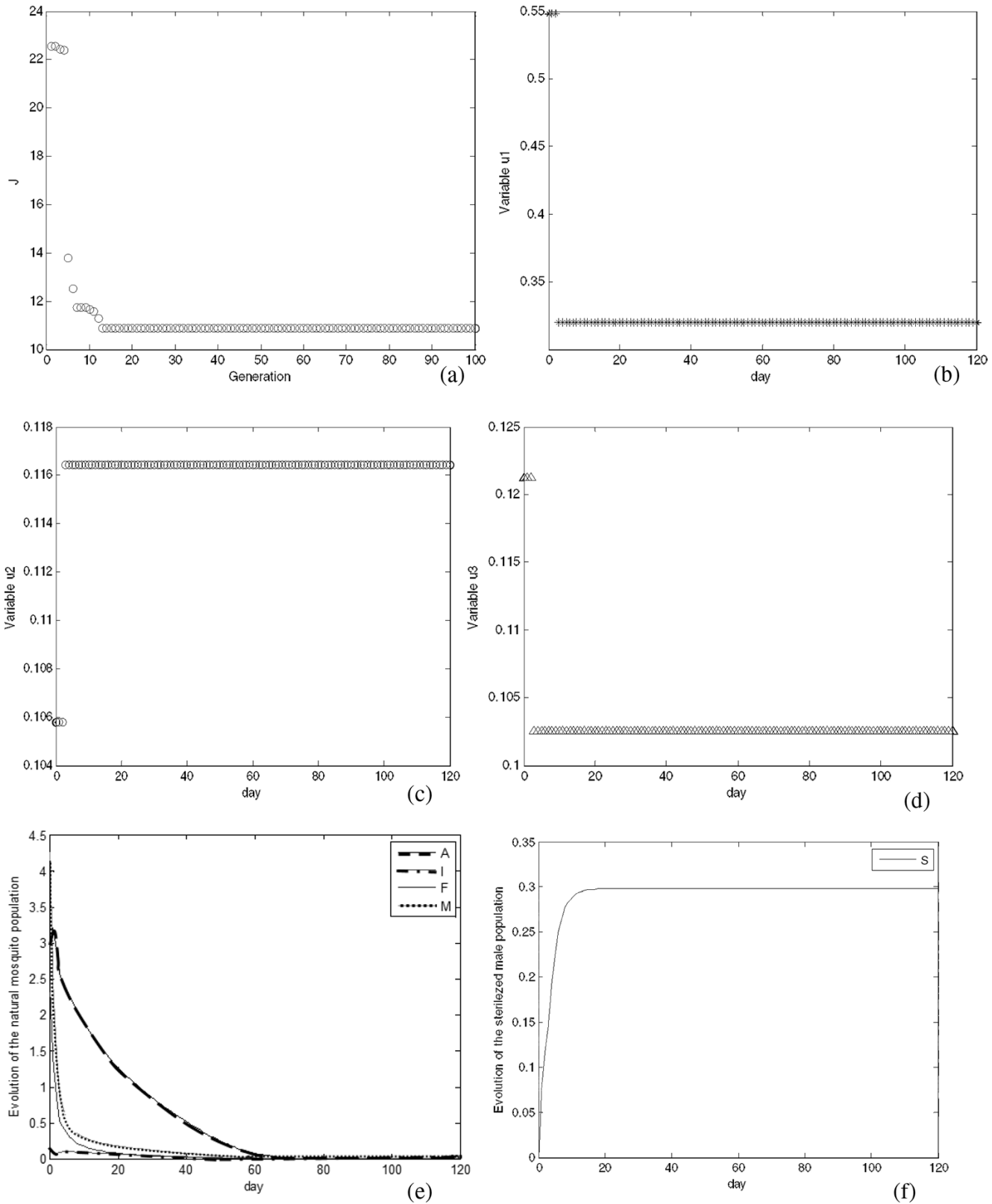

Figure 11 - Results of GA_AEDES algorithm when using $u_{1}, u_{2}$ and $u_{3}$ controls: (a) objective function value along the generations; (b), (c) and (d) contain, respectively, the best $u_{1}, u_{2}$ and $u_{3}$ controls; (e) evolution of natural mosquito population - segments $M, F, I$ and $A$; (f) evolution of sterilized mosquito population $S$. 
Table 3 - Characteristics of the additional set of test instances.

\begin{tabular}{c|c|l}
\hline Instance & $\begin{array}{c}\text { Control } \\
\text { application } \\
\text { time }\end{array}$ & \multicolumn{1}{|c}{ Instance information } \\
\hline $\mathrm{I}_{60}$ & 60 days & Applying control only 60 days in the early summer \\
$\mathrm{I}_{130}$ & 130 days & Applying control in the first 130 days of the year (whole summer) \\
$\mathrm{I}_{180}$ & 180 days & Applying control in the first 180 days of the year \\
$\mathrm{I}_{365}$ & 365 days & Applying control throughout the year \\
\hline No control & 365 days & No control was applied during the year \\
\hline
\end{tabular}

Figure 12 presents the results of the application of the algorithm AG_AEDES with instances I $_{60}$, $\mathrm{I}_{130}, \mathrm{I}_{180}, \mathrm{I}_{365}$ and No control (Table 3 ).
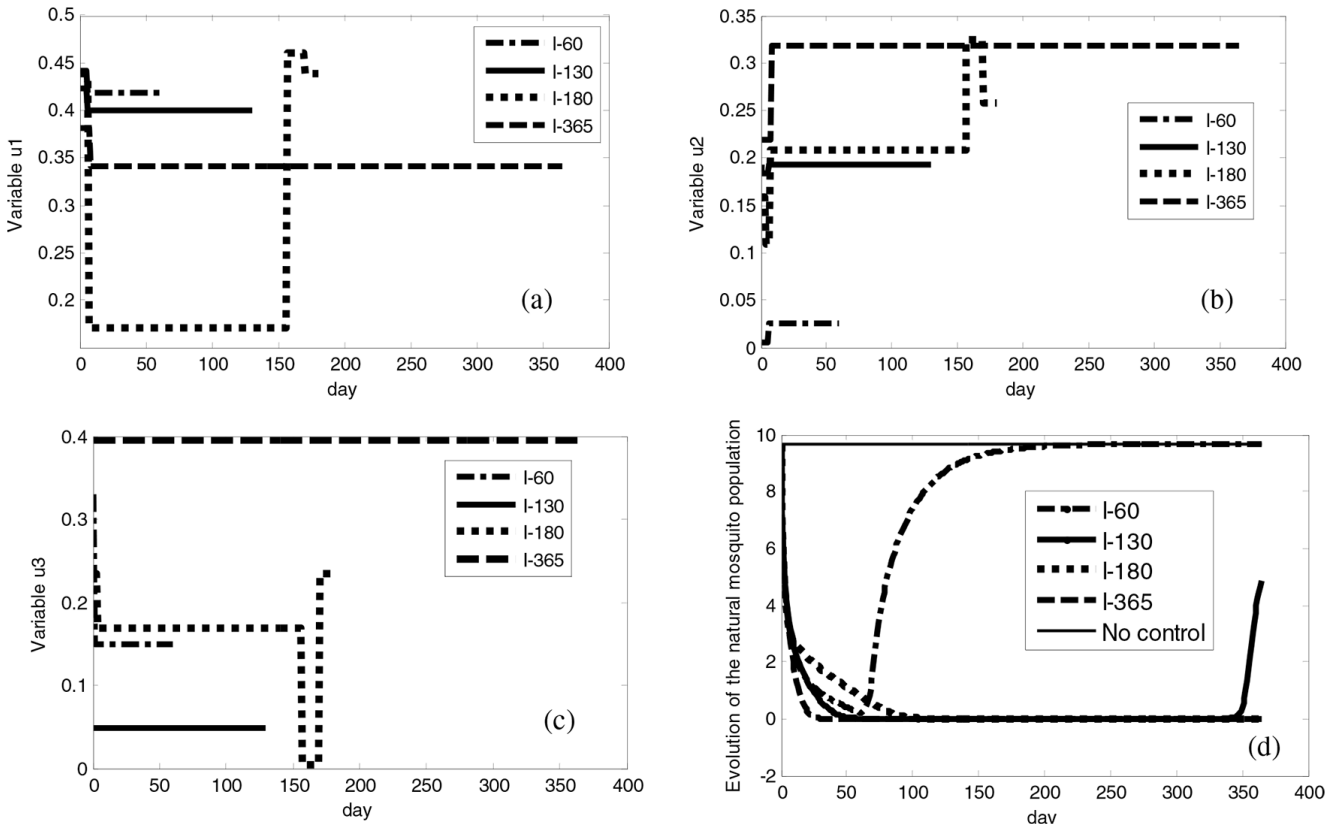

Figure 12 - Results of GA_AEDES algorithm when using $u_{1}, u_{2}$ and $u_{3}$ controls in instances $\mathrm{I}_{60}, \mathrm{I}_{130}$, $\mathrm{I}_{180}$ and $\mathrm{I}_{365}$ (Table 3): (a), (b) and (c) contain, respectively, the optimized $u_{1}, u_{2}$ and $u_{3}$ controls for all instances; (d) evolution of natural mosquito population - sum of the segments $M, F, I$ and $A$ for all instances.

GA_AEDES has determined good quality strategies for instances $\mathrm{I}_{60}, \mathrm{I}_{130}, \mathrm{I}_{180}$ and $\mathrm{I}_{365}$, as shown in Figure 12. A comparison of chemical, physical and biological control techniques are presented in Figure 12 (a), (b) and (c). In Figure 12 (d) we can observe that when the control is applied for a short period, shorter than the length of the summer period $\left(I_{60}\right)$, the population of mosquitos 
grows rapidly. When the application of the control lasts for 130 days, stopping soon after the end of summer $\left(\mathrm{I}_{130}\right)$, the mosquito population starts growing again just before the summer of the next year. In all other cases, when the control is applied for six months or more, it is possible to determine strategies that maintain the mosquito population at low levels throughout the year. The population remains at high levels towards the whole year when controls are not applied.

This computational experiments show that the algorithm GA_AEDES enables the study of good quality strategies for combined chemical, biological and physical controls. Health care decision makers plan the control applications for pre-determined discretized time intervals, hence the approach used in this paper is more appropriate for real life problems than time continuous approaches, namely the one presented by Thomé et al. [17]. The results of this study also indicate that the inclusion of a constraint on the state variable $F$ provides controls with better performance, when compared with the ones obtained by Thomé et al. [17]. In fact, in the experiments performed by these authors, the mosquito populations in the different phases (aquatic, immature, fertilized females and males), even when optimal control is acting, start increasing after the 96th day. This does not happen with the genetic methodology presented in this paper.

\section{CONCLUSION}

Dengue, Chikungunya and Zika are diseases that have raised worries within the public health systems in tropical countries throughout the world. All efforts made to control these diseases have therefore been well accepted. In this research work, a genetic algorithm has been implemented to determine good quality feasible solutions for an aggregated objective optimization problem proposed to control Aedes mosquitoes' population by means of chemical, biological and physical procedures. Here, a new procedure, physical control, is added to the other controls already considered in previous studies. The problem tackles the solution quality by considering an optimization objective that aggregates five sub-objectives.

The computational experiments with real data instances of the problem studied for 60, 120, 130, 180 and 365-day periods revealed the power of the algorithm to determine feasible solutions at a low computational effort, mainly as a result of the penalization technique for inclusion of constraints.

A significant differential of this work is the possibility of establishing optimal strategies for the mosquito control using an integration of different types of controls, including physical control, which is the most environmentally friendly.

The results show that the chemical control with insecticides is very efficient in order to reduce the amount of mosquitoes as compared with two other discussed controls, but the intense and constant use of chemicals can cause serious damage to the environment, and to human and animal health. However, the results indicate that the use of chemical control combined with other types of controls promotes reducing the amount of insecticide needed. This study also shows that a combination of chemical, biological and physical controls significantly reduces the disease hazard. In particular, it shows that with a reasonably low effort from each control, harmful natural 
mosquitoes, from all segments, can be extinguished during the period of control application. Thereby, this methodology can assist policy makers in planning the optimal use of combined chemical, physical and biological controls.

A possible future extension is the investigation of mathematical models that incorporate resistance of mosquitoes due to insecticide.

\section{ACKNOWLEDGEMENTS}

We wish to thank FAPESP (Grant No. 2009/15098-0 [Temático], No. 2009/14901-4, No. 2010/07585-6 and 2014/01604-0), FUNDUNESP/PROPE/UNESP (0351/019/13), CNPq (302454/2016-0), CAPES and PROPG UNESP for their financial support. Margarida Vaz Pato's research was supported by Portuguese national funding from Fundação para a Ciência e a Tecnologia under project POCTI-ISFL-1-152.

\section{REFERENCES}

[1] Aldila D, Götz T \& Soewono E. 2013. An optimal control problem arising from a dengue disease transmission model. Mathematical Biosciences, 242: 9-16. doi .org/10 .1016/j . mbs . 2012.11 .014

[2] Dias WO, Wanner EF \& CARdoso RTN. 2015. A multiobjective optimization approach for combating Aedes aegypti using chemical and biological alternated step-size control. Mathematical Biosciences, 269: 37-47.

[3] Esteva L \& YAng H. 2005. Mathematical model to assess the control of Aedes aegypti mosquitoes by the Sterile Insect Technique. Mathematical Biosciences, 198: 132-147. doi: 10.1016/j.mbs.2005.06.004

[4] Florentino HO, Bannwart BF, Cantane DR \& Santos FLP. 2014. Multiobjective genetic algorithm applied to dengue control. Mathematical Biosciences, 258: 77-84. doi .org/10 .1016/ j.mbs.2014.08.013

[5] Gerald C \& Wheatley P. 2003. Applied Numerical Analysis. Addison-Wesley Publishing Company, New York.

[6] Ghosh M, Lashari AA \& Li XZ. 2013. Biological control of malaria: A mathematical model. Applied Mathematics and Computation, 219: 7923-7939. Doi: 10.3390/toxins4090748.

[7] Goldberg DE. 2002. Genetic Algorithms and Evolutionary Computation. Academic Publishers, Norwell.

[8] GoRdiLlo LF. 2013. Reducing mosquito-borne disease outbreak size: The relative importance of contact and transmissibility in a network model. Applied Mathematical Modelling, 37: 8610-8616. doi.org/10.1016/j.apm.2013.03.056

[9] KNIPLING EF. 1985. Sterile insect technique as a screwworm control measure: The concept and its development. Miscellaneous Publications of the Entomological Society of America, 62: 4-7.

[10] Koiller MaS, Souza MO, Codeço C, Iggidr A \& Sallet G. 2014. Aedes, Wolbachia and Dengue. INRIA Research Report RR8462, 2014. https: / / hal . archives-ouvertes.fr/ hal-00939411/document 
[11] MATLAB. 2012. Version 7.14.0.739 (R2012a). Documentation Center. The MathWorks Inc, 2012. http : / / uk . mathworks . com/help/search. html ?qdoc $=r 2012 \mathrm{a}$

[12] Manyombe MlM, Tsanou B, Mbang J \& Bowong S. 2017. A metapopulation model for the population dynamics of Anopheles mosquito. Applied Mathematics and Computation, 307: 71-91. doi: 10.1016/j.amc.2017.02.039.

[13] Mishra A \& GaKKhar S. 2014. The effects of awareness and vector control on two strains dengue dynamics. Applied Mathematics and Computation, 246: 159-167. doi: 10.1016/j.amc.2014.07.115

[14] Mustafa MS, Rasotgi V, Jain S \& Gupta V. 2015. Discovery of fifth serotype of dengue virus (DENV-5): A new public health dilemma in dengue control. Medical Journal Armed Forces India, 71: 67-70. doi.org/10.1016/j.mjafi.2014.09.011

[15] Pates H \& CURTIS C. 2005. Mosquito behavior and vector control. Annual Review of Entomology, 50: 53-70. doi.org/10.1146/annurev .ento.50.071803.130439

[16] Rodrigues HS, Monteiro MTT \& Torres DFM. 2010. Dynamics of dengue epidemics when using optimal control. Mathematical and Computer Modelling, 52: 1667-1673. doi.org/10 . $1016 / j . m c m .2010 .06 .034$

[17] Thomé RCA, YAng HM \& Esteva L. 2010. Optimal control of Aedes aegypti mosquitoes by the sterile insect technique and insecticide. Mathematical Biosciences, 223: 12-23. doi .org/10. $1016 / j$.mbs. 2009.08 .009

[18] Vasilakis N, Cardosa J, Hanley Ka, Holmes EC \& Weaver SC. 2011. Fever from the forest: prospects for the continued emergence of sylvatic dengue virus and its impact on public health. Nature Reviews Microbiology, 9: 532-541. doi: 10.1038/nrmicro2595

[19] Whitley D. 1994. A genetic algorithm tutorial. Statistics and Computing, 4: 65-85. doi:10.1007/BF00175354.

[20] WhO - World Health Organization, Dengue. 2016. Available at: http: / www . who. int/mediacentre/factsheets/fs117/en/. Accessed July 13, 2016.

[21] Wijaya KP, GöTz T \& Soewono E. 2014. An optimal control model of mosquito reduction management in a dengue endemic region. International Journal of Biomathematics, 7: 1-22. doi.org/10.1142/S1793524514500569.

[22] YANG HM \& Ferreira CP. 2008. Assessing the effects of vector control on dengue transmission. Applied Mathematics and Computation. 198: 401-413. doi: 10.1016/j.amc.2007.08.046

[23] Yang HM, Macoris MLG, Galvani KC, Andrighetti MTM \& Wanderley DMV. 2009. Assessing the effects of temperature on the population of Aedes aegypti, vector of dengue. Epidemiology \& Infection. 137: 1188-1202. doi: 10.1017/S0950268809002040.

[24] Zhang H, Georgescu P \& Hassan AS. 2016. Mathematical insights and integrated strategies for the control of Aedes aegypti. Applied Mathematics and Computation, 273: 1059-1089. doi .org/ $10.1016 / j$.amc.2015.10.066.

[25] Zhang Q \& Li H. 2007. A Multiobjective Evolutionary Algorithm Based on Decomposition. IEEE Transactions on Evolutionary Computation, 11: 712-731. doi: 10.1109/TEVC.2007.892759. 